\title{
Comments
}

\section{Concerning the Paper ,Horseshoes in Perturbations of Hamiltonian Systems with Two Degrees of Freedom" by P. J. Holmes and J. E. Marsden}

\author{
S. L. Ziglin \\ Institute of Radio Engeneering and Electronics, USSR Academy of Sciences, SU-103907 Moscow, \\ Marx Avenue, 18, USSR
}

In Holmes and Marsden (1982) the Hamiltonian systems with two degrees of freedom depending on a small parameter and integrable for the value of a parameter equal to zero were considered. It was noted that when the unperturbed problem has no homoclinic orbits and the averaged perturbed system has such orbits the splitting of separatrices is exponentially small with respect to the small parameter. Thus the proper consideration of the problem must take into account the terms of the order of smallness higher than one and contain a careful analysis of errors. It was stated also that the restricted problem of four point vortices considered in Ziglin (1980) belongs to the same class of problems. Later, on the basis of this statement, in Koiller and de Carvahlo (1983) the conclusion was made that the consideration of the restricted problem of four point vortices in Ziglin (1980), taking into account only the terms of the first order with respect to the small parameter, is not correct.

It must be noted in this connection that the restricted problem of four point vortices does not belong to the class of problems considered by Holmes and Marsden. In this problem the homoclinic orbits exist already in the unperturbed system and the splitting of separatrices has the first order with respect to the small parameter. So the conclusions made in Holmes and Marsden (1982) are not applicable to this problem. Since the mentioned statement of Holmes and Marsden is repeatedly cited (see e.g. [Aref (1985)]), I thought it was necessary to clarify this question.

\section{References}

Aref, H.: Dynamics of a few vortices. In: Theoretical and applied mechanics. Niordson, F., Olhoff, N. (eds.), pp. 43-68. Amsterdam: North-Holland 1985

Holmes, P.J., Marsden, J.E.: Horseshoes in perturbations of Hamiltonian systems with two degrees of freedom. Commun. Math. Phys. 82, 523-544 (1982) 
Koiller, J., de Carvahlo, S.P.: Nonintegrability of a restricted problem of four point vortices: informes de matematica pura e aplicada, Serie A-022-Juhlo/83. Rio de Janeiro 1983; Chaos and nonintegrability of point Vortex motions. To appear in J. Fluid Mech.

Ziglin, S.L.: Nonintegrability of a problem on the motion of four point vortices. Sov. Math. Dokl. 21, 296-299 (1980)

Communicated by A. Jaffe

Received June 12, 1986 